\title{
OFF-STREET PARKING FACILITY LOCATION ON URBAN TRANSPORTATION NETWORK CONSIDERING MULTIPLE OBJECTIVES: A CASE STUDY OF ISFAHAN (IRAN)
}

\author{
Mahdi ESKANDARI ${ }^{*}$, Ali Shahandeh NOOKABADI ${ }^{1,2}$ \\ ${ }^{1}$ Toos Institute of Higher Education, Mashhad, Iran \\ ${ }^{2}$ Dept of Industrial and Systems Engineering, Isfahan University of Technology, Iran \\ Received 4 January 2016; revised 16 April 2016; accepted 3 June 2016; \\ published online 4 September 2017
}

\begin{abstract}
The increasing population in large cities and the unbalanced urban growth associated with massive use of private cars in metropolitan areas often lead to traffic jams and road congestion that warrant the construction of such capital-intensive buildings as off-street public parking facilities. However, the initial problem in such projects is locating a suitable spot where all citizens can be conveniently served and the traffic load in busy city centers can be reduced by removing the need for on-street parking facilities. In this paper, an urban transportation network including, a number of parking demand points, a set of possible sitting locations, and several entry points of traffic flow are considered. Four objectives are generally considered for the public off-street parking location problem that include reducing traffic congestion, maximizing coverage demand, minimizing walking distance between demand points and new parking facilities, and decreasing related costs. The flow-capturing model has been exploited to develop two approaches for minimizing traffic congestion. Based on these approaches and other objectives, two models have been proposed. The covering distance of parking facilities will be uncertain in these models. Traffic flow entry points, driver's path, and different types of parking lots have also been considered. Finally, relevant information and data required for implementing the proposed models were collected on two traffic zones in Central Business Districts (CBD) of Isfahan (Iran). Then, the $\varepsilon$-constraint method was used to solve the proposed multi-objectives models and the best candidate points for establishing new off-street parking facilities were determined.
\end{abstract}

Keywords: facility location, public parking lots, off-street parking facility, flow-capturing, multi-objective decisionmaking, mathematical modelling.

\section{Introduction}

Traffic congestion is a major problem in metropolitan cities. It is being aggravated by such factors as growing urbanization, concentration of activities in Central Business Districts (CBD), population influx, and poor public transport systems. This is while problems such reducing street capacity due to on-street parking, irregular and slow movement of cars searching for vacant car park spaces, and delays caused by entering or leaving a car park in the street leads to even more traffic jams and road congestion. One of the negative effects that can be associated to parking in urban region is the presence of cruising traffic. That is, drivers may need to drive around whereas searching for a vacant parking spot. This leads to additional traffic on the urban network (Chaniotakis, Pel 2015). Construction of off-street parking lots seems to offer one possible solution to the situation.
Such facilities naturally need to be erected in the right location in order to ensure that the objectives justifying the investment are achieved and that the citizens are properly served. As in all other public projects, there are different stakeholders such as the local government, investors, and users. Hence, a solution is required that satisfies all the stakeholders. Another question that must be addressed is the selection of the right off-street parking lot from among the different surface, multi-story, underground, roofed, and mechanical types (Shahi 2011). Each has its own costs and provides a different capacity. Thus, the type of parking lot forms a decisionmaking parameter. The main objective of the present study is to determine the best location and the most appropriate type of new off-street parking facilities to achieve maximally reduced traffic congestion, to provide

${ }^{\star}$ Corresponding author. E-mail: m.eskandari@in.iut.ac.ir 
maximum coverage at demand points, and to minimize the walking distances among the facilities at the lowest total costs. In Section 2, previous studies of the off-street parking location problem are reviewed. In Section 3, the assumptions of the problem and the models proposed will be presented. Section 4 will describe the data collection method, implementation of the models, and the results obtained from the implementing proposed models for two different traffic zones of CBD's in Isfahan (Iran) as a case study. Finally, conclusions and suggestions will be presented in the last Section.

\section{Literature review}

Facility location has of long been considered as an important decision-making problem. However, the formal study of facility location started in 1909 when Weber studied the location of a warehouse such that all the distances between the warehouse and various customers were minimized (Drezner, Hamacher 2002). The facility location theory has ever since been employed and different models have been developed with various applications in different fields. The models thus far developed can be classified from different aspects but they are generally are classified into discrete, continuous, and network-based models, based on their solution spaces. Eight basic models may be exploited for locating a given facility on a network; they include: set covering, maximal covering, $P$-center, $P$-dispersion, $P$-median, fixed charge, hub and maxisum (Drezner, Hamacher 2002). One important aspect of urban transportation system that plays an important role in decreasing traffic congestion is off-street parking facilities. Many studies have been conducted on parking facilities locations some of which are based on Geographic Information Systems (GIS) and Analytical Hierarchy Process (AHP).

Ghanbari and Ghazi Asgar (2011) identified the following five criteria for determining parking lot location: distance from absorbtion centers, proximity to busy streets, suitable land use for parking, value of property, density of inhabitants. They calculated the weight of each criterion by pairwise comparison and tested different methods of compound overlay including the Boolean, multiple weighting, and fuzzy logic. In next stage, they prepared a utility map using each of these methods and found that the fuzzy logic provided the best results. Jelokhani-Niaraki and Malczewski (2015a) in their study presented a Web-based group GIS-MCDA approach to address the issue of parking lot location in Tehran. They claimed that the integration of GIS and Multi-Criteria Decision Analysis (MCDA) capabilities into the Web platform has offered an effective MultiCriteria Spatial Decision Support System (MC-SDSS) with which to involve different groups in parking lot selection processes. They believed the system makes it possible to find appropriate locations that may reconcile the various and conflicting objectives resulting from different views and the final site selection outcome that can be generally accepted.
Aliniai et al. (2015) in their study aim at finding the most suitable sites for public parking lots considered five criteria of 'distance from travel absorber centers', 'distance from passages', 'the cost of real estate' and 'suitable land uses for parking lots', while 'unsuitable land uses for public parking spaces' such as historical places were regarded as a constraint factor and excluded from being further analysed. Using pair wise comparisons the selected criteria were weighted and then by applying Boolean Method and fuzzy Ordered Weighted Average (OWA) the map layers were finally overlaid. The other GIS-based studies on parking location adopted the same methodology but only changed the criteria or the layers combining procedures (Tang et al. 2013; He et al. 2015; Jelokhani-Niaraki, Malczewski 2015b).

A number of studies adopted the mathematical modelling approach. Dirickx and Jennergren (1975) used an assignment model for determining the optimum allocation of existing parking spaces with different types of parking demand. Goyal and Gomez (1984) proposed a linear programming model for determining the optimum allocation of campus car parking facilities to different classes of users. Wey (2003) assumed that parking demand changes over a given time horizon and $P$ new parking lots have to be located at given times. The problem is to find the best location for the new parking facilities.

Chiu (2005) considered two objective functions. The first is to maximize parking demand fulfilled by parking facilities and the second is to minimize the total social costs which include construction costs, operating and maintenance costs for operators, walking cost for users, the costs of reducing air and noise pollution covered by all people, and the penalty cost for the unfulfilled demands. Wang et al. (2008) in their study tried to determine the best locations for public parking facilities with the objective of minimizing both the total cost and the weighted distance between the parking facilities and demand points. Here, the number of new parking facilities is unknown but efforts are made to satisfy the total parking demand with the minimum number of parking lots.

Most previous studies have used one objective function. However, multiple objectives are preferred due to the nature of the public parking location problem and the requirement to satisfy various stakeholders' demands. In addition to these parameters, the present study will also include flow entry points, driver's route, and the existing parking lots that have been neglected in most previous studies on the parking facility location problem. Moreover, most parameters have been generally assumed to be deterministic, which will receive a non-deterministic treatment in this study.

\section{Problem definition and modelling}

In this research, it is assumed that part of the urban transportation network includes a number of parking demand points (places where attract trips) and a number of candidate points for locating parking lots where cars arrive from different points and routes. It is further assumed that cars enter the area from different points 
(called flow entry points) at a certain or pre-specified rate. In addition, there is a possibility to locate any type of parking lot at any candidate point but only at varying costs and with different capacities depending on the parking type selected. Since the distance of parking lot for different demand points are not the same, in this paper the coverage distance considered uncertain. If the distance between demand point and a parking lot is less than $D c_{1}$, then the car drivers willing to go to the parking lot. As this distance increases, the likelihood of people to use this car park is reduced. If the distance is longer than $D c_{2}$, in this case the car drivers reluctant to go to the parking lot.

In addition to these assumptions, the following assumptions are considered in this study:

- the distance between a demand point and a candidate point is calculated based on the walking distance.

- the distance between flow entry points and candidate points and that between flow entry points and demand points are calculated based on the distance travelled by car.

- the distance between various points are considered equal to the shortest path between them on the network and are calculated based on the distance between the centers of gravity.

- a number of parking facilities already exist in the region.

\subsection{Notations used}

The notations for this problem are defined as follows.

\subsubsection{Parameters}

$I$ - the set of parking demand points $i$;

$J$ - the set of parking facilities $j$;

$K$ - the set of flow entry points indexed by $k$;

$P$ - the set of parking facilities types indexed by $p$;

$d_{i j}$ - the distance between demand point $i$ and candidate point $j$;

$d_{k j}^{\prime}$ - the distance between entry point $k$ and candidate point $j$;

$d_{k i}^{\prime \prime}$ - the distance between entry point $k$ and demand point $i$;

$\theta_{i j}$ - if demand point $i$ is located within the maximum coverage distance of candidate point $j, 1$; otherwise, 0 ;

$u_{i j}$ - utility of candidate point $j$ for demand point $i$;

$P d_{k i}$ - the amount of parking demand at point $i$ of entry point $k$;

$b_{j p}$ - the capacity at candidate point $j$ if type $p$ is selected (maximum number of cars that can be parked in this type of facility);

$C c_{j p}$ - the cost of property acquisition and facility constructing at candidate point $j$ if type $p$ is selected;

$O M c_{p}$ - annual operation and maintenance cost for each unit of type $p$;

$P c$ - annual penalty cost per unit of unsatisfied demand;
$D c_{1}$ - lower bound of coverage distance;

$D c_{2}$ - upper bound of coverage distance;

$n$ - number of new off-street parking facilities;

$M$ - a large constant;

$M f$ - another large constant.

\subsubsection{Decision variables}

$y_{j p}$ - if a parking type $P$ is located at candidate point $j, 1$; otherwise, 0 ;

$x_{k i j p}$ - the amount of parking demand from entry point $k$ to demand point $i$ served by parking type $p$ located at candidate point $j$;

$z_{k i}$ - the unsatisfied demand at point $i$ of entry point $k$;

$v_{j p}$ - the free capacity at candidate point $j$ if parking type $p$ is located.

\subsection{Objective functions}

In this paper, four objectives are considered for the public parking location problem. The first involves minimizing traffic congestion. The second and third are combined together and meant to maximize the demand covered and minimize the distance walked. Finally, the fourth objective is to minimize different costs.

\subsubsection{Minimizing traffic congestion}

One of the objective functions in the parking location problem is minimizing the traffic volume in the region. For this purpose, it is necessary to consider the entry points of traffic flow to the region. This objective can be achieved through two approaches.

\subsubsection{First approach}

This approach aims to minimize traffic congestion by minimizing the travel distance between flow entry points and new parking lots. Equation (1) shows the objective function of minimizing traffic congestion based on this approach:

$$
\begin{aligned}
& \text { Minimize } Z_{1}=\sum_{k \in K} \sum_{i \in I} \sum_{j \in J} \sum_{p \in P}\left(d_{k j}^{\prime} \cdot \theta_{i j} \cdot x_{k i j p}+\right. \\
& \left.M \cdot\left(1-\theta_{i j}\right) \cdot x_{k i j p}\right)+\sum_{k \in K} \sum_{i \in I} M f \cdot z_{k i} .
\end{aligned}
$$

The first part of this equation is the product of the distance between flow entry points and new parking lots in the demand served by these parking lots. It should be noted that this part of the objective function is only applied for demand points and the candidate points located within the maximum coverage distance (otherwise, $\theta_{i j}$ is equal to zero). The second part ensures that if a candidate point $j$ does not cover the demand point $i, x_{k i j p}$ should be zero.

The last term in this equation forces the model to reduce the amount of unsatisfied demand; in other words, the amount of traffic congestion caused by unsatisfied demands will be minimized. The objective function is expressed in number of cars at travelled distance (the volume of traffic). 


\subsubsection{Second approach}

This approach seeks to maximize the distance between demand points and new parking lots in order to reduce the volume of traffic in the region. Put differently, it tries to maximize the flow capture from the network, that is, drivers can find a parking lot on their way to their points of destination (Khakbaz et al. 2013).

Based on this approach, the objective function of maximizing traffic congestion reduction can be written as in Equation (2). Given that the distance between demand points and new parking lots depends on different points of flow entry, it will be necessary to consider traffic flow entry points. In Equation (2), the product of distances between demand points and new parking facilities in the amount of parking demand between these points is expressed:

$$
\text { Maximize } Z_{1}=\sum_{k \in K} \sum_{i \in I} \sum_{j \in J} \sum_{p \in P}\left(d_{k i}^{\prime \prime}-d_{k j}^{\prime}\right) \cdot \theta_{i j} \cdot x_{k i j p} \text {. }
$$

This objective function aims at maximizing the distance between demand points and new parking lots, but the coefficient $\theta_{i j}$ in Equation (2) ensures that these points do not exceed the upper bound of coverage distance. Like the previous approach, this objective function is expressed in number of cars in travelled distance.

\subsubsection{Maximizing coverage demand and minimizing walking distance}

One of the objectives considered for enhancing the productivity of off-street parking facilities is maximizing the coverage demand (Chiu 2005). Minimizing the walking distance is another objective that has attracted much attention (Ghanbari, Ghazi Asgar 2011; Wey 2003; Chiu 2005; Wang et al. 2008). In this study, these two functions are considered together while it is assumed that the coverage distance is uncertain.

A utility criterion $u_{i j}$ based on the distance between demand points and candidate points is defined between zero and one. In this research, two coverage distances are considered. If the distance between the demand point and the candidate point is less than the lower bound of coverage distance $D c_{1}$, the utility criterion is equal to one. The value decreases as the distance increases until the distance exceeds the upper bound of coverage distance $D c_{2}$ when the value for the criterion will be equal to zero. Equation (3) calculates the value for this criterion:

$$
u_{i j}= \begin{cases}1, & d_{i j} \leq D c_{1} ; \\ f\left(d_{i j}\right), & D c_{1} \leq d_{i j} \leq D c_{2} ; \\ 0, & D c_{2} \leq d_{i j} .\end{cases}
$$

Assuming that $f\left(d_{i j}\right)$ is calculated by Equation (4), Figure 1 shows $u_{i j}$ on the basis of different distances between demand and candidate points:

$$
f\left(d_{i j}\right)=\frac{D c_{2}-d_{i j}}{D c_{2}-D c_{1}} .
$$

According to the utility criterion defined above, the objective function of maximizing coverage demand is

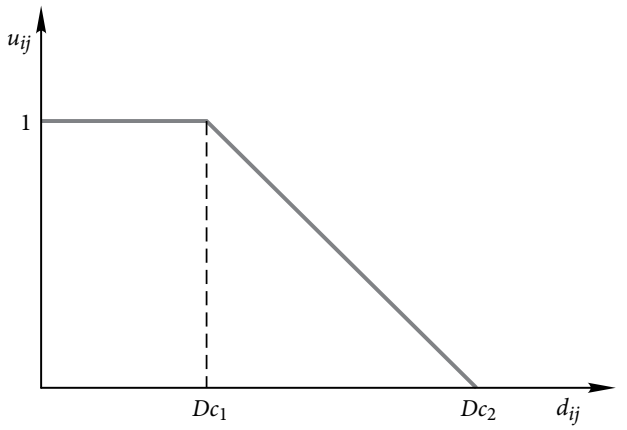

Figure 1. Utility criterion based on the distance between demand and candidate points

given by Equation (5) in which, the parking coverage distance is assumed to be uncertain. Other purpose of this equation is to minimize the walking distance between parking facilities and demand points. This function is expressed in demand value:

$$
\text { Maximize } Z_{2}=\sum_{k \in K} \sum_{i \in I} \sum_{j \in J} \sum_{p \in P} u_{i j} \cdot x_{k i j p} .
$$

\subsubsection{Minimizing different costs}

Another objective is minimizing the costs as shown in Equation (6):

$$
\begin{aligned}
& \text { Minimize } Z_{3}=\sum_{j \in J} \sum_{p \in P}\left(C c_{j p}+O M c_{p} \cdot b_{j p}\right) \times \\
& y_{j p}+\sum_{k \in K} \sum_{i \in I} P c \cdot z_{k i} .
\end{aligned}
$$

The first component of the objective function captures the costs of property acquisition, facility construction, and annual operation and maintenance. The second component expresses the penalty cost for unsatisfied demands. In order to express these different components in the same unit, costs are calculated on an annual basis. Assuming that the parking fee is based on the total costs per unit of car park, minimizing the value for this criterion will minimize the parking fee.

\subsection{Proposed models}

Based on the different approaches cited above and considering the objectives described, two multi-objective models are proposed which are named MOPLP1 (Multi-Objective Parking Location Problem1) and MOPLP2. The first objective functions in these two models are not identical.

\subsubsection{MOPLP1 model}

This model is based on the first approach as follows:

$$
\begin{aligned}
& \text { Minimize } Z_{1}=\sum_{k \in K} \sum_{i \in I} \sum_{j \in J} \sum_{p \in P}\left(d_{k j}^{\prime} \cdot \theta_{i j} \cdot x_{k i j p}+\right. \\
& \left.M \cdot\left(1-\theta_{i j}\right) \cdot x_{k i j p}\right)+\sum_{k \in K} \sum_{i \in I} M f \cdot z_{k i} ; \\
& \text { Maximize } Z_{2}=\sum_{k \in K} \sum_{i \in I} \sum_{j \in J} \sum_{p \in P} u_{i j} \cdot x_{k i j p} ;
\end{aligned}
$$




$$
\begin{aligned}
& \text { Minimize } Z_{3}=\sum_{j \in J} \sum_{p \in P}\left(C c_{j p}+O M c_{p} \cdot b_{j p}\right) \times \\
& y_{j p}+\sum_{k \in K} \sum_{i \in I} P c \cdot z_{k i},
\end{aligned}
$$

subject to:

$$
\begin{aligned}
& \sum_{j \in J} \sum_{p \in P} x_{k i j p}+z_{k i}=P d_{k i}, \forall k \in K, \quad \forall i \in I ; \\
& \sum_{k \in K} \sum_{i \in I} x_{k i j p}+v_{j p}=b_{j p} \cdot y_{j p}, \forall j \in J, \quad \forall p \in P ; \\
& \sum_{p \in P} y_{j p} \leq 1, \forall j \in J ; \\
& \sum_{j \in J} \sum_{p \in P} y_{j p}=n ; \\
& y_{j p} \in\{0,1\}, \quad \forall j \in J, \quad \forall p \in P ; \\
& x_{k i j p} \geq 0, \quad \forall k \in K, \quad \forall i \in I, \quad \forall j \in J, \quad \forall p \in P ; \\
& z_{k i} \geq 0, \quad \forall k \in K, \quad \forall i \in I ; \\
& v_{j p} \geq 0, \forall j \in J, \forall p \in P .
\end{aligned}
$$

Constraint (7) ensures that the parking demand at any demand point is either served by a facility or considered as an unsatisfied demand. Constraint (8) determines the total demand assigned to each facility and insures that the allocated demand to each candidate point is not greater than its capacity. Constraint (9) indicates that one type of parking lot can only be deployed at any candidate point. Constraint (10) states the number of new off-street parking facilities to be built. Relations (11)-(14) represent decision variables of the model.

To take into account the existing parking lots in proposed model, they are assumed to be candidate points which have been located before. It is also necessary that the number of existing parking lots is added to the number of new parking lots, while the costs of land acquisition and construction for the existing parking facilities are taken to be zero.

\subsubsection{MOPLP2 model}

This model has been developed based on the second approach:

$$
\begin{aligned}
& \text { Maximize } Z_{1}=\sum_{k \in K} \sum_{i \in I} \sum_{j \in J} \sum_{p \in P}\left(d_{k i}^{\prime \prime}-d_{k j}^{\prime}\right) \cdot \theta_{i j} \cdot x_{k i j p} \\
& \text { Maximize } Z_{2}=\sum_{k \in K} \sum_{i \in I} \sum_{j \in J} \sum_{p \in P} u_{i j} \cdot x_{k i j p} \\
& \text { Minimize } Z_{3}=\sum_{j \in J} \sum_{p \in P}\left(C c_{j p}+O M c_{p} \cdot b_{j p}\right) \times \\
& y_{j p}+\sum_{k \in K} \sum_{i \in I} P c \cdot z_{k i}, \\
& \text { subject to: } \\
& \sum_{j \in J} \sum_{p \in P} x_{k i j p}+z_{k i}=P d_{k i}, \quad \forall k \in K, \quad \forall i \in I \\
& \sum_{k \in K} \sum_{i \in I} x_{k i j p}+v_{j p}=b_{j p} \cdot y_{j p}, \quad \forall j \in J, \quad \forall p \in P
\end{aligned}
$$

$$
\begin{aligned}
& \sum_{p \in P} y_{j p} \leq 1, \quad \forall j \in J ; \\
& \sum_{j \in J} \sum_{p \in P} y_{j p}=n ; \\
& y_{j p} \in\{0,1\}, \quad \forall j \in J, \quad \forall p \in P ; \\
& x_{k i j p} \geq 0, \quad \forall k \in K, \quad \forall i \in I, \quad \forall j \in J, \quad \forall p \in P ; \\
& z_{k i} \geq 0, \quad \forall k \in K, \quad \forall i \in I ; \\
& v_{j p} \geq 0, \quad \forall j \in J, \quad \forall p \in P .
\end{aligned}
$$

\section{Case study}

Isfahan is the third largest city in Iran. It is the center of Isfahan Province and due to its historical, cultural, and industrial attractions has a relatively high population growth. The city is currently experiencing a lot of traffic problems. One of these is the lack of enough parking spaces in its busy central region. A recent study on Isfahan transportation system reported that Isfahan consists of 321 traffic zones including 181 internal zones, 131 external zones, and 9 link zones (Dehnavi et al. 2013). The traffic zones 1 to 12 are part of the internal zones, which are located in the CBD of the city. The historical centers, several administrative and service buildings, and major marketplaces in these zones have always been the source of traffic problems in these centers. Figure 2 depicts this problematic area.

Table 1 and Figure 3 show the average quantity of parking spaces lacking in these zones for different hours of the day (Eskandari 2012). As shown in Figure 3, zone 12 has the highest shortage of parking spaces followed by zones 4, 5, 6 and 7 . The peak hour for the observed shortage of parking spaces is different in different traffic zones.

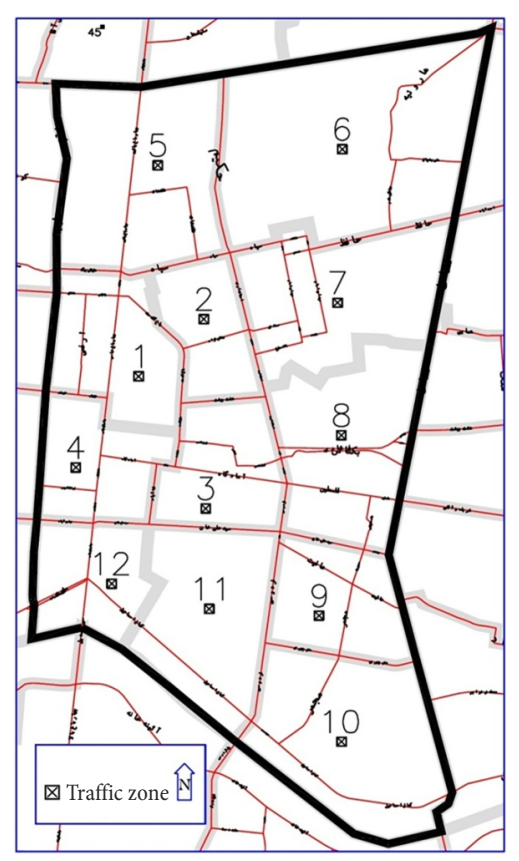

Figure 2. Traffic zones 1 to 12 in Isfahan 
Table 1. Average parking space lacking in each traffic zone at different hours on each day of the week (Eskandari 2012)

\begin{tabular}{|c|c|c|c|c|c|c|c|c|c|c|c|c|}
\hline \multirow{2}{*}{ Hour } & \multicolumn{12}{|c|}{ Traffic zones } \\
\hline & 1 & 2 & 3 & 4 & 5 & 6 & 7 & 8 & 9 & 10 & 11 & 12 \\
\hline 8 & 0 & 0 & 33 & 0 & 331 & 0 & 121 & 0 & 243 & 0 & 0 & 780 \\
\hline 9 & 137 & 0 & 245 & 0 & 863 & 1118 & 832 & 0 & 450 & 0 & 0 & 1690 \\
\hline 10 & 269 & 0 & 452 & 78 & 1160 & 1596 & 1078 & 0 & 481 & 0 & 0 & 2140 \\
\hline 11 & 285 & 0 & 392 & 205 & 1328 & 1556 & 1024 & 0 & 480 & 0 & 0 & 2043 \\
\hline 12 & 167 & 0 & 205 & 0 & 986 & 1026 & 738 & 0 & 320 & 0 & 0 & 1613 \\
\hline 13 & 0 & 0 & 0 & 0 & 384 & 68 & 240 & 0 & 211 & 0 & 0 & 1082 \\
\hline 14 & 0 & 0 & 0 & 0 & 176 & 0 & 0 & 0 & 0 & 0 & 0 & 720 \\
\hline 15 & 0 & 0 & 69 & 0 & 825 & 0 & 4 & 0 & 37 & 0 & 0 & 838 \\
\hline 16 & 0 & 0 & 328 & 0 & 1199 & 477 & 388 & 0 & 70 & 0 & 0 & 1613 \\
\hline 17 & 197 & 0 & 574 & 677 & 1262 & 849 & 749 & 0 & 191 & 0 & 0 & 2503 \\
\hline 18 & 205 & 0 & 587 & 968 & 1062 & 806 & 694 & 0 & 123 & 0 & 0 & 2500 \\
\hline 19 & 102 & 0 & 412 & 1282 & 815 & 240 & 516 & 0 & 84 & 0 & 0 & 2304 \\
\hline 20 & 0 & 0 & 0 & 454 & 391 & 0 & 29 & 0 & 0 & 0 & 0 & 1799 \\
\hline 21 & 0 & 0 & 0 & 0 & 0 & 0 & 0 & 0 & 0 & 0 & 0 & 260 \\
\hline
\end{tabular}

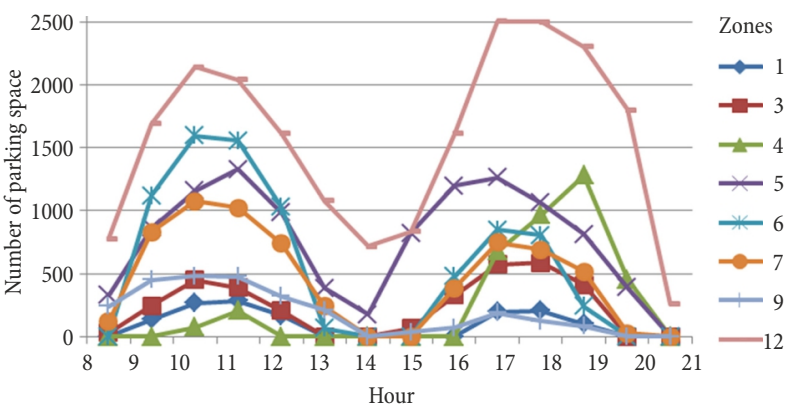

Figure 3. Average parking space lacking in each traffic zone at different hours on each day of the week (Eskandari 2012)

Depending on the nature of each traffic zone, the peak hour for some zones occurs in the morning but for others it occurs in the evening. In this study, zones 4 and 12 were considered as the study area for the location of off-street parking lots due to their proximity and their high shortage of parking spaces.

\subsection{Data collection}

In order to implement the models for locating parking lots in the study area, the required data were gathered.

Considering the urban development plan of Isfahan which in it the number of parking spaces required for different land uses has been separately specified (Naghsh e Jahan-Pars 2008), a number was assigned to each demand point, these numbers were then normalized (divided by their sum) and thus, the weights for the demand points were determined. In the next step, the demand for these points were calculated by multiplying the peak hour parking demands (zones 4 and 12) times the weights of the demand points (Eskandari 2012).

\subsubsection{Demand points}

To determine the demand points, the different land uses and urban facilities existing in a neighbourhood were clas- sified based on distance and area, and each category was considered as a demand point. A view of the study area and demand points in this region is shown in Figure 4.

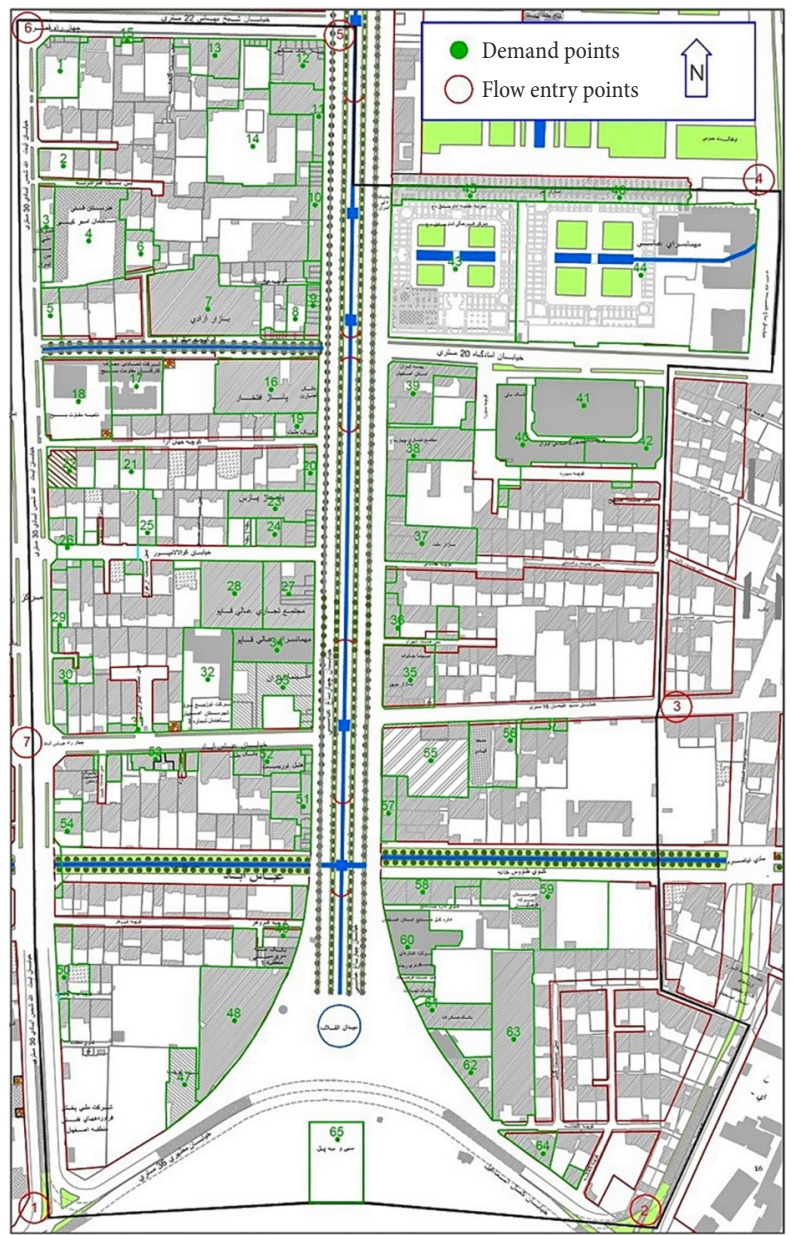

Figure 4. Parking demand points and flow entry points in the study area 


\subsubsection{Flow entry points}

For the purposes of this study, it is assumed that the traffic flows into the study area from various entry points (origins of traffic flow). Given the existing paths, 7 flow entry points were identified for this area. In Figure 4 shows the entry points of traffic flow in the study area. Using origin-destination information, the number of cars entering the study area was determined. Then, by conducting a field study, the ratio of entering cars from each entry point was identified. The demands of entry points was calculated by multiplying these ratios with the number of cars entering the study area (Eskandari 2012).

\subsubsection{Candidate points and available parking lots}

In this study, candidate points were selected for building parking lots based on the detailed development plan of Isfahan while some were also recommended by the Transportation and Traffic Department (TTD) of Isfahan Municipal Government. Ultimately, 11 candidate points were determined. In the next step, the existing public parking lots in the study area were identified. Table 2 shows the details on these parking lots.

The candidate and existing parking lots in the study area are shown in Figure 5 in which candidate points are

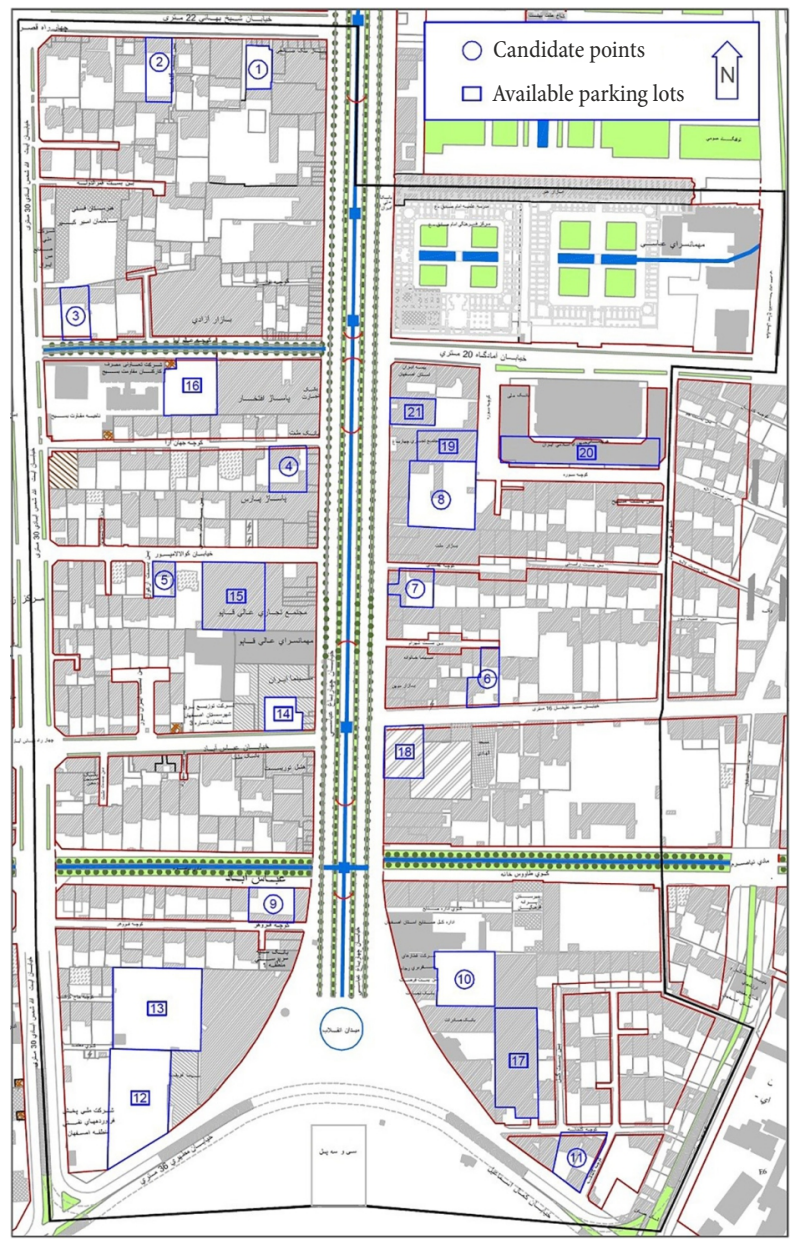

Figure 5. Candidate points and existing parking lots in the study area designated by numbers 1 to 11 and locations of existing parking lots by numbers 12 to 21 . Among the existing parking lots, three (namely, Siyosepol, Enghelab and Abas-Abad designated by numbers 12, 13 and 14) are of the surface type that may be changed to other types of parking lot.

Three types of surface, multi-story, and mechanical parking lots are considered for construction in the study area. In each candidate point, one type of parking lot can be constructed. Table 3 shows the required space per unit for different parking lot types (Tehran Barnamerizi Shahri 2006). The capacity of each candidate point for each type of parking facility was determined based on the assumption that the maximum floor number allowed in the study area is 5 .

To determine the utility criterion and the value of $\theta_{i j}$, the detailed development plan of Isfahan was considered and the upper and lower bounds of coverage distance were taken to be 300 and 150 meters, respectively (Naghsh e Jahan-Pars 2008). Furthermore, the various car park costs for each parking type were determined using expert views of TTD. It is also assumed that the annual penalty cost per unit of unsatisfied demand $(P c)$ is equal to $250 \mathrm{EUR}$. In this case study, the number of variables and constraints is 29246 (including 63 binary and 29183 continuous variables) and 530, respectively.

Table 2. Existing parking lots in the study area

\begin{tabular}{|c|l|l|c|}
\hline No & \multicolumn{1}{|c|}{ Parking name } & \multicolumn{1}{c|}{ Type } & Capacity \\
\hline 12 & Siyosepol & Surface & 140 \\
\hline 13 & Enghelab & Surface & 170 \\
\hline 14 & Abas-Abad & Surface & 30 \\
\hline 15 & Ali Qapu & Underground & 100 \\
\hline 16 & Eftekhar & Multi-story & 320 \\
\hline 17 & Sepahan Complex & Underground & 80 \\
\hline 18 & City Center & Underground & 90 \\
\hline 19 & Chahar Bagh & Underground & 40 \\
\hline 20 & Abasi Complex & Underground & 100 \\
\hline 21 & Ferdosi Complex & Underground & 35 \\
\hline
\end{tabular}

Table 3. The required space per unit of each parking type (Tehran Barnamerizi Shahri 2006)

\begin{tabular}{|c|l|c|}
\hline$P$ & Parking type & Required area $\left[\mathrm{m}^{2}\right]$ \\
\hline 1 & Surface parking & 25 \\
\hline 2 & Multi-story parking & $30-35$ \\
\hline 3 & Mechanical parking & $20-25$ \\
\hline
\end{tabular}

\section{Model solution}

\subsection{The single-objective problem}

The GAMS 22.1 software and CPLEX solver were used for solving the MOPLP1 model to obtain the ideal solution for each objective function (Rosenthal 2016). The results are presented in Tables 4-6, where in these tables $Z_{1}, Z_{2}$ and $Z_{3}$ are in kilometers, number of cars, and thousand EUR per year, respectively. Since the optimum 
solutions of the single-objective functions of MOPLP1 are not the same, the optimal orientations of the three objective functions may be said to be different. It will, therefore, be necessary to use multi-objective decision making methods for solving the problem and to select the best answer obtained.

The results obtained from solving the single-objective MOPLP2 are shown in Tables 7-9. Clearly, the optimal solutions are not the same in this case, either. It follows then that Multi-Objective Decision-Making (MODM) methods should be used for solving the model.

\subsection{MODM methods}

MODM methods are concerned with mathematical optimization problems involving more than one objective function to be optimized simultaneously. In contrast to single-objective optimization problems, a Multi-objective Optimization Problem (MOP) may have not just one, but many optimal solutions. Due to the many and often competing objectives in a MOP, there are several trade-off solutions, which are optimal in the sense that there is no better solution for any of the objective functions simultaneously. These optimal solutions are called Pareto-optimal solutions. MODM methods are classified into four classes according to the decision maker's intervention (Figueira et al. 2005):

- methods without the decision maker's intervention;

- methods in which basic information are obtained from the decision maker beforehand;

- interactive methods;

- methods in which the decision maker's opinion are applied after problem solving.

From another point of view, MODM methods can be divided into two general classes including: methods leading to satisfactory solutions and those leading to efficient solutions. In this study, the $\varepsilon$-constraint method is utilized. It has been proved that the unique solution
Table 4. Single-objective optimal solving of MOPLP1 by first objective for every $n$

\begin{tabular}{|c|c|c|c|}
\hline$n$ & $Z_{1}^{*}$ & $Z_{2}$ & $Z_{3}$ \\
\hline 0 & 2745 & 1702 & 1322 \\
\hline 1 & 2482 & 1856 & 1565 \\
\hline 2 & 2331 & 2056 & 1870 \\
\hline 3 & 2188 & 2275 & 2000 \\
\hline 4 & 2106 & 2040 & 1824 \\
\hline 5 & 2000 & 2146 & 1827 \\
\hline 6 & 1929 & 2185 & 1935 \\
\hline 7 & 1908 & 2174 & 1980 \\
\hline 8 & 1801 & 2425 & 2334 \\
\hline 9 & 1771 & 2438 & 2406 \\
\hline 10 & 1731 & 2529 & 2555 \\
\hline 11 & 1726 & 2415 & 2416 \\
\hline
\end{tabular}

Table 7. Single-objective optimal solving of MOPLP2 by first objective for every $n$

\begin{tabular}{|c|c|c|c|}
\hline$n$ & $Z_{1}^{*}$ & $Z_{2}$ & $Z_{3}$ \\
\hline 0 & 329.6 & 940 & 1535 \\
\hline 1 & 444.7 & 1120 & 1393 \\
\hline 2 & 452.6 & 1121 & 1439 \\
\hline 3 & 499.5 & 1185 & 1583 \\
\hline 4 & 554.4 & 1424 & 1830 \\
\hline 5 & 584.2 & 1482 & 1961 \\
\hline 6 & 603.7 & 1560 & 2045 \\
\hline 7 & 620.8 & 1596 & 2191 \\
\hline 8 & 630.9 & 1644 & 2253 \\
\hline 9 & 645.5 & 1753 & 2379 \\
\hline 10 & 647.4 & 1756 & 2455 \\
\hline 11 & 650.5 & 1755 & 2843 \\
\hline
\end{tabular}

Table 5. Single-objective optimal solving of MOPLP1 by second objective for every $n$

\begin{tabular}{|c|c|c|c|}
\hline$n$ & $Z_{1}$ & $Z_{2}^{*}$ & $Z_{3}$ \\
\hline 0 & 3022 & 2010 & 1322 \\
\hline 1 & 3143 & 2197 & 1232 \\
\hline 2 & 2980 & 2459 & 1390 \\
\hline 3 & 2810 & 2808 & 2095 \\
\hline 4 & 2830 & 2878 & 1648 \\
\hline 5 & 2728 & 3092 & 1955 \\
\hline 6 & 2671 & 3249 & 1875 \\
\hline 7 & 2608 & 3428 & 1983 \\
\hline 8 & 2568 & 3594 & 2103 \\
\hline 9 & 2576 & 3672 & 2162 \\
\hline 10 & 2528 & 3729 & 2252 \\
\hline 11 & 2473 & 3704 & 2337 \\
\hline
\end{tabular}

Table 8. Single-objective optimal solving of MOPLP2 by second objective for every $n$

\begin{tabular}{|c|c|c|c|}
\hline$n$ & $Z_{1}$ & $Z_{2}^{*}$ & $Z_{3}$ \\
\hline 0 & 0 & 2010 & 1322 \\
\hline 1 & 0 & 2259 & 1275 \\
\hline 2 & 0 & 2563 & 1651 \\
\hline 3 & 910 & 2808 & 1951 \\
\hline 4 & 374 & 2940 & 1691 \\
\hline 5 & 0 & 3171 & 2022 \\
\hline 6 & 0 & 3152 & 1849 \\
\hline 7 & 0 & 3485 & 2025 \\
\hline 8 & 0 & 3625 & 2146 \\
\hline 9 & 0 & 3699 & 2205 \\
\hline 10 & 0 & 3740 & 2312 \\
\hline 11 & 0 & 3716 & 2390 \\
\hline
\end{tabular}

Table 6. Single-objective optimal solving of MOPLP1 by third objective for every $n$

\begin{tabular}{|c|c|c|c|}
\hline$n$ & $Z_{1}$ & $Z_{2}$ & $Z_{3}^{*}$ \\
\hline 0 & 77226 & 258 & 719 \\
\hline 1 & 86435 & 162 & 751 \\
\hline 2 & 68264 & 342 & 797 \\
\hline 3 & 98053 & 153 & 855 \\
\hline 4 & 91390 & 244 & 916 \\
\hline 5 & 66650 & 396 & 978 \\
\hline 6 & 89110 & 245 & 1043 \\
\hline 7 & 103270 & 161 & 1112 \\
\hline 8 & 96654 & 300 & 1182 \\
\hline 9 & 99999 & 328 & 1267 \\
\hline 10 & 107840 & 314 & 1398 \\
\hline 11 & 112780 & 340 & 1546 \\
\hline
\end{tabular}

Table 9. Single-objective optimal solving of MOPLP2 by third objective for every $n$

\begin{tabular}{|c|c|c|c|}
\hline$n$ & $Z_{1}$ & $Z_{2}$ & $Z_{3}^{*}$ \\
\hline 0 & 0 & 226 & 719 \\
\hline 1 & 0 & 295 & 751 \\
\hline 2 & 0 & 230 & 797 \\
\hline 3 & 0 & 231 & 855 \\
\hline 4 & 0 & 207 & 916 \\
\hline 5 & 0 & 252 & 978 \\
\hline 6 & 0 & 392 & 1043 \\
\hline 7 & 0 & 253 & 1112 \\
\hline 8 & 0 & 341 & 1182 \\
\hline 9 & 0 & 121 & 1267 \\
\hline 10 & 0 & 346 & 1398 \\
\hline 11 & 0 & 315 & 1546 \\
\hline
\end{tabular}


of this method is an efficient solution (Ehrgott 2005). It is also worth mentioning that the decision maker's comments are applied after solving the problem.

\subsection{The $\varepsilon$-constraint method}

Consider the following Multi-Objective Mathematical Programming (MOMP) problem:

$$
\text { Maximize }\left\{f_{j}(x), j=1, \ldots, p\right\} \text {, }
$$

subject to:

$x \in S$,

where: $x$ is the vector of decision variables; $f_{j}(x)$ are the objective functions; $S$ is the feasible area. In the $\varepsilon$-constraint method, one of the objective functions is optimized and the other objectives are considered as constraints, incorporating them in the constraint part of the model as shown below (Mavrotas 2009):

Maximize $f_{i}(x)$,

subject to:

$$
\begin{aligned}
& f_{j}(x) \leq \varepsilon_{j}, \quad j=1, \ldots, p, \quad j \neq i ; \\
& x \in S .
\end{aligned}
$$

By effecting changes in the RHS of the constrained objective functions $\varepsilon_{j}$, the various efficient solutions of the problem are obtained. Eventually, after enough efficient solutions have been generated, the decision maker selects the preferred solution based on the weights assigned to various objective functions. The $\varepsilon$-constraint method has several advantages including the ability to generate different efficient solutions from various parts of the solution space as well, no need for scaling the objective functions, and simplicity. Its shortcomings include the efficiency of the optimal solution obtained is not guaranteed and the ranges of the objective functions should be specified in order to generate the grid points that will act as RHS (Nosoohi, Hejazi 2011).

\subsubsection{Implementation of the MOPLP1 model}

In order to implement the $\varepsilon$-constraint method, the first objective function (minimizing traffic congestion) was chosen as the objective function and the other objectives were added to the constraints. Equation (17) shows how this method is implemented for the MOPLP1 model.

Minimize $Z_{1}$,

subject to:

$$
\begin{aligned}
& Z_{2} \geq \varepsilon_{2} ; \\
& Z_{3} \leq \varepsilon_{3} ; \\
& x \in S .
\end{aligned}
$$

The model was solved in GAMS 22.1 using CPLEX solver in a $2.13 \mathrm{GHz}, 1 \mathrm{~GB}$ RAM machine. Ten efficient solutions were obtained for $n=0$ (the number of new off- street parking equals to zero) and by different values of $\varepsilon_{j}$ as shown in Table 10 where, the values for the objective functions $\varepsilon_{j}$ and the type of existing parking facilities are also shown for each of the efficient solutions (Rosenthal 2017). As can be seen, no new parking facilities are required but the type of the existing ones have changed as per the solutions obtained.

Table 10 is like a decision-making matrix in which rows represent alternatives while the second, third, and fourth columns represent different criteria. Each of the matrix elements is called $r_{i j}$.

Table 10. Efficient solutions for MOPLP1 using the $\varepsilon$-constraint method when $n=0$

\begin{tabular}{|c|c|c|c|c|c|c|c|c|}
\hline $\begin{array}{l}\text { Efficient } \\
\text { solution }\end{array}$ & $Z_{1}$ & $Z_{2}$ & $Z_{3}$ & $\varepsilon_{2}$ & $\varepsilon_{3}$ & \multicolumn{3}{|c|}{$\begin{array}{c}\text { Location, type) } \\
\text { of existing } \\
\text { parking lots }\end{array}$} \\
\hline 1 & 2745 & 1687 & 1322 & 1500 & 1375 & $(12,3)$ & $(13,3)$ & $(14,3)$ \\
\hline 2 & 2745 & 1800 & 1178 & 1800 & 1250 & $(12,2)$ & $(13,3)$ & $(14,3)$ \\
\hline 3 & 2875 & 1800 & 1032 & 1800 & 1125 & $(12,1)$ & $(13,3)$ & $(14,3)$ \\
\hline 4 & 3048 & 1800 & 990 & 1800 & 1000 & $(12,3)$ & $(13,1)$ & $(14,3)$ \\
\hline 5 & 3034 & 1100 & 860 & 1100 & 875 & $(12,2)$ & $(13,1)$ & $(14,1)$ \\
\hline 6 & 3320 & 1100 & 719 & 1100 & 750 & $(12,1)$ & $(13,1)$ & $(14,1)$ \\
\hline 7 & 2922 & 1500 & 931 & 1500 & 950 & $(12,1)$ & $(13,2)$ & $(14,3)$ \\
\hline 8 & 2980 & 1600 & 931 & 1600 & 1000 & $(12,1)$ & $(13,2)$ & $(14,3)$ \\
\hline 9 & 2895 & 1400 & 931 & 1400 & 1000 & $(12,1)$ & $(13,2)$ & $(14,3)$ \\
\hline 10 & 2914 & 1950 & 1072 & 1950 & 1125 & $(12,2)$ & $(13,2)$ & $(14,3)$ \\
\hline
\end{tabular}

The fuzzy dimensionless method is represented by Equation (18) for a positive index (like the second objective function) and by Equation (19) for a negative indicator (such as the first and third objective functions). The measurement scale in this method ranges between zero and one such that zero represents the worst and one represents the best answer (Tzeng, Huang 2011). The results of this dimensionless method are presented is Table 11.

$$
\begin{aligned}
n_{i j} & =\frac{r_{i j}-\min _{i} r_{i j}}{\max _{i} r_{i j}-\min _{i} r_{i j}} ; \\
n_{i j} & =\frac{\max _{i} r_{i j}-r_{i j}}{\max _{i} r_{i j}-\min _{i} r_{i j}},
\end{aligned}
$$

where: $n_{i j}$ - normalized element of $r_{i j}$.

To select the best solution among the efficient ones, AHP was used as shown in Figure 6. Assuming the same weights for the different objectives (indices) and using the Expert Choice software, the weight of each efficient solution for the off-street parking location was obtained. Based on the results shown in Table 12, the tenth efficient solution was found to be the best (preferred) solution.

In a similar manner, the preferred solutions for other values of $n(n=1, \ldots, 11)$ can be obtained for different numbers of new parking facilities. Table 13 shows the preferred solutions selected for different values of $n$ in the MOPLP1 model (the model took approximately $20 \mathrm{~s}$ to run). As can be seen, the multi-story or mechanical type has often been selected for new parking facilities since surface parking lots are not economical to construct in the city center. 


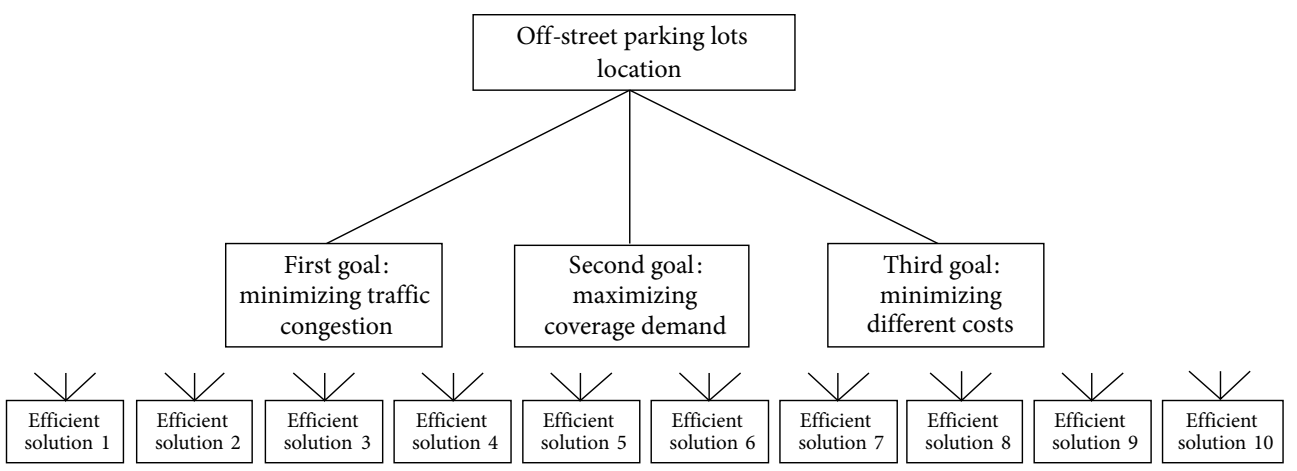

Figure 6. Hierarchical structure

Table 11. Dimensionless decision making matrix for MOPLP1 based on $n=0$

\begin{tabular}{|c|c|c|c|}
\hline Efficient solution & $Z_{1}$ & $Z_{2}$ & $Z_{3}$ \\
\hline 1 & 1.000 & 0.691 & 0.000 \\
\hline 2 & 1.000 & 0.824 & 0.240 \\
\hline 3 & 0.774 & 0.824 & 0.481 \\
\hline 4 & 0.473 & 0.824 & 0.551 \\
\hline 5 & 0.497 & 0.000 & 0.766 \\
\hline 6 & 0.000 & 0.000 & 1.000 \\
\hline 7 & 0.692 & 0.471 & 0.649 \\
\hline 8 & 0.591 & 0.588 & 0.649 \\
\hline 9 & 0.739 & 0.353 & 0.649 \\
\hline 10 & 0.706 & 1.000 & 0.414 \\
\hline
\end{tabular}

Table 12. Weights of efficient solutions for the parking lot location in the MOPLP1 model $(n=0)$

\begin{tabular}{|c|c|c|c|c|c|c|c|c|c|c|}
\hline \multirow{2}{*}{ Goal } & \multicolumn{7}{|c|}{ Efficient solutions } \\
\cline { 2 - 10 } & 1 & 2 & 3 & 4 & 5 & 6 & 7 & 8 & 9 & 10 \\
\hline $\begin{array}{l}\text { Parking } \\
\text { location }\end{array}$ & 0.097 & 0.118 & 0.119 & 0.106 & 0.072 & 0.057 & 0.104 & 0.105 & 0.100 & 0.121 \\
\hline
\end{tabular}

Clearly, the mechanical type has been proposed for small values of $n$ but the multi-story type is often selected for higher values of $n$. The reason for this is that the shortage of parking spaces in the study area will be high for small values of $n$ so that parking lots with high capacities such as the mechanical type will be needed. This shortage reduces with increasing values of $n$. Therefore, the need for high capacity parking lots that also warrant high costs - will be reduced. Using this model, the prioritized candidate points were determined as presented in Table 13.

\subsubsection{Implementation of MOPLP2 model}

In a fashion similar to what went above on MOPLP1, the preferred solutions may be obtained by the MOPLP2 model based on different numbers of new parking facilities. Table 14 presents the preferred solutions that are finally selected by the MOPLP2 model based on different values of $\mathrm{n}$. Due to the differences between the approaches adopted for reducing traffic congestion, the process of prioritizing candidate points will be somewhat different in the two models.

Table 13. The preferred solutions selected by MOPLP1 based on different values of $n$

\begin{tabular}{|c|c|c|c|c|c|c|c|c|c|c|c|c|c|c|c|c|c|}
\hline$n$ & $Z_{1}$ & $Z_{2}$ & $Z_{3}$ & \multicolumn{11}{|c|}{ (Location, type) of the new parking lots } & \multicolumn{3}{|c|}{$\begin{array}{l}\text { (Location, type) of } \\
\text { existing parking lots }\end{array}$} \\
\hline 0 & 2914 & 1950 & 1072 & & & & & & & & & & & & $(12,2)$ & $(13,2)$ & $(14,3)$ \\
\hline 1 & 2730 & 2180 & 1230 & $(11,3)$ & & & & & & & & & & & $(12,2)$ & $(13,2)$ & $(14,3)$ \\
\hline 2 & 2639 & 2400 & 1357 & $(11,3)$ & $(2,3)$ & & & & & & & & & & $(12,2)$ & $(13,2)$ & $(14,3)$ \\
\hline 3 & 2533 & 2600 & 1468 & $(11,3)$ & $(2,2)$ & $(10,2)$ & & & & & & & & & $(12,2)$ & $(13,2)$ & $(14,3)$ \\
\hline 4 & 2363 & 2875 & 1691 & $(11,3)$ & $(2,3)$ & $(10,3)$ & $(4,3)$ & & & & & & & & $(12,1)$ & $(13,3)$ & $(14,3)$ \\
\hline 5 & 2226 & 3000 & 1804 & $(11,3)$ & $(2,3)$ & $(10,3)$ & $(4,3)$ & $(6,3)$ & & & & & & & $(12,1)$ & $(13,3)$ & $(14,3)$ \\
\hline 6 & 2349 & 3200 & 1825 & $(11,3)$ & $(2,3)$ & $(10,2)$ & $(4,3)$ & $(6,3)$ & $(7,3)$ & & & & & & $(12,1)$ & $(13,3)$ & $(14,3)$ \\
\hline 7 & 2179 & 3000 & 1758 & $(11,2)$ & $(1,3)$ & $(10,2)$ & $(4,3)$ & $(6,2)$ & $(7,3)$ & $(3,2)$ & & & & & $(12,1)$ & $(13,3)$ & $(14,3)$ \\
\hline 8 & 2186 & 3200 & 1843 & $(11,3)$ & $(2,2)$ & $(10,2)$ & $(4,2)$ & $(6,2)$ & $(7,3)$ & $(3,2)$ & $(9,2)$ & & & & $(12,1)$ & $(13,3)$ & $(14,3)$ \\
\hline 9 & 2140 & 3000 & 1762 & $(11,2)$ & $(2,2)$ & $(10,2)$ & $(4,2)$ & $(6,2)$ & $(7,2)$ & $(3,2)$ & $(1,2)$ & $(5,2)$ & & & $(12,1)$ & $(13,3)$ & $(14,3)$ \\
\hline 10 & 1874 & 2800 & 1926 & $(11,2)$ & $(2,2)$ & $(10,3)$ & $(4,2)$ & $(6,2)$ & $(7,2)$ & $(3,2)$ & $(9,2)$ & $(5,2)$ & $(1,2)$ & & $(12,1)$ & $(13,3)$ & $(14,3)$ \\
\hline 11 & 2026 & 3000 & 1915 & $(11,2)$ & $(2,1)$ & $(10,2)$ & $(4,2)$ & $(6,2)$ & $(7,2)$ & $(3,2)$ & $(9,2)$ & $(5,2)$ & $(1,2)$ & $(8,1)$ & $(12,1)$ & $(13,2)$ & $(14,3)$ \\
\hline
\end{tabular}


Table 14. The preferred solutions selected by MOPLP2 based on different values of $n$

\begin{tabular}{|c|c|c|c|c|c|c|c|c|c|c|c|c|c|c|c|c|c|}
\hline$n$ & $Z_{1}$ & $Z_{2}$ & \multicolumn{10}{|c|}{$Z_{3}$} & \multicolumn{10}{|c|}{ (Location, type) of the new parking lots } & \multicolumn{3}{|c|}{$\begin{array}{c}\text { Location, type) of } \\
\text { existing parking lots }\end{array}$} \\
\hline 0 & 270 & 1500 & 1016 & & & & & & & & & & & & $(12,1)$ & $(13,3)$ & $(14,2)$ \\
\hline 1 & 369 & 1500 & 1087 & $(10,2)$ & & & & & & & & & & & $(12,1)$ & $(13,2)$ & $(14,3)$ \\
\hline 2 & 365 & 1900 & 1275 & $(10,3)$ & $(11,2)$ & & & & & & & & & & $(12,1)$ & $(13,2)$ & $(14,3)$ \\
\hline 3 & 402 & 2000 & 1359 & $(10,2)$ & $(11,2)$ & $(7,2)$ & & & & & & & & & $(12,1)$ & $(13,3)$ & $(14,3)$ \\
\hline 4 & 438 & 2100 & 1465 & $(10,3)$ & $(11,2)$ & $(7,2)$ & $(9,3)$ & & & & & & & & $(12,1)$ & $(13,2)$ & $(14,3)$ \\
\hline 5 & 427 & 2200 & 1476 & $(10,2)$ & $(11,3)$ & $(7,2)$ & $(9,2)$ & $(4,2)$ & & & & & & & $(12,1)$ & $(13,2)$ & $(14,3)$ \\
\hline 6 & 508 & 2000 & 1549 & $(10,3)$ & $(11,2)$ & $(7,2)$ & $(9,2)$ & $(1,2)$ & $(6,2)$ & & & & & & $(12,1)$ & $(13,2)$ & $(14,3)$ \\
\hline 7 & 472 & 2100 & 1499 & $(10,2)$ & $(11,2)$ & $(7,2)$ & $(9,2)$ & $(1,2)$ & $(6,2)$ & $(5,2)$ & & & & & $(12,1)$ & $(13,2)$ & $(14,3)$ \\
\hline 8 & 479 & 2500 & 1714 & $(10,3)$ & $(11,2)$ & $(7,2)$ & $(9,3)$ & $(4,2)$ & $(6,2)$ & $(5,2)$ & $(1,2)$ & & & & $(12,1)$ & $(13,2)$ & $(14,3)$ \\
\hline 9 & 436 & 2600 & 1750 & $(10,3)$ & $(11,2)$ & $(7,2)$ & $(2,2)$ & $(4,2)$ & $(6,2)$ & $(5,2)$ & $(1,2)$ & $(3,2)$ & & & $(12,1)$ & $(13,2)$ & $(14,3)$ \\
\hline 10 & 440 & 2800 & 1869 & $(10,2)$ & $(11,2)$ & $(7,2)$ & $(9,3)$ & $(4,2)$ & $(6,1)$ & $(5,2)$ & $(1,2)$ & $(3,2)$ & $(2,2)$ & & $(12,1)$ & $(13,3)$ & $(14,2)$ \\
\hline 11 & 479 & 2500 & 1870 & $(10,2)$ & $(11,2)$ & $(7,2)$ & $(9,2)$ & $(4,2)$ & $(6,1)$ & $(5,2)$ & $(1,2)$ & $(3,2)$ & $(2,1)$ & $(8,1)$ & $(12,1)$ & $(13,2)$ & $(14,2)$ \\
\hline
\end{tabular}

\section{Conclusions}

In this study, the location theory was employed to investigate the public parking lot location problem. In addition, the multi-objective mathematical programming was used to satisfy the stakeholders by the different objectives. The objectives considered here included minimizing traffic congestion, maximizing the coverage demand, and minimizing the walking distances and different costs.

For reducing traffic congestion, two different approaches were proposed and the flow entry points and vehicle paths were taken into account. The first approach was based on the distance between entry points and new parking facilities while the second was based on the distance between demand points and new parking facilities. Based on these two approaches, two different models were developed. The objective functions of maximizing covered demand and minimizing walking distances were combined and the coverage distance of the parking facilities was assumed to be uncertain. Due to differences in capacity and costs associated with each parking lot type, different types of parking facilities were considered.

To evaluate the models, their performance was investigated in the CBD of Isfahan (Iran). The two traffic zones 4 and 12 located in the central district that suffer from shortage of parking spaces were selected as the study area for implementation of the models. The required data were collected for both zones. The $\varepsilon$-constraint method was then used to solve the proposed multi-objective models and the preferred solution among the efficient solutions generated was selected based on the AHP technique. The results indicate the location and type of new parking facilities, the best type of parking lot for the existing facilities, and the quantity of satisfied demand.

It was assumed that the utility of each candidate point from the perspective of demand points is the distance between the points whereas this utility criterion can also be related to other issues such as demand level, the capacity of the candidate points, parking fees, etc. For further study, the actual value of this utility can be more exactly determined by properly identifying the factors affecting this criterion. Another area for future research is considering different coverage distances based on different trip objectives. Most of the parameters used in this problem have been assumed to be given. For a more realistic problem, stochastic values and fuzzy variables may be used to develop new models.

\section{Acknowledgements}

The authors would like to thank the Iran National Science Foundation (INSF) for partially financially supporting this research under Contract No. INSF 87041478.

The authors also appreciate the cooperation lent to them by the Traffic and Transportation Department of Isfahan Municipality (Iran).

\section{References}

Aliniai, K.; Yarahmadi, A.; Zarin, J. Z.; Yarahmadi, H.; Lak, S. B. 2015. Parking lot site selection: an opening gate towards sustainable GIS-based urban traffic management, Journal of the Indian Society of Remote Sensing 43(4): 801813. https://doi.org/10.1007/s12524-014-0415-3

Chaniotakis, E.; Pel, A. J. 2015. Drivers' parking location choice under uncertain parking availability and search times: a stated preference experiment, Transportation Research Part A: Policy and Practice 82: 228-239. https://doi.org/10.1016/j.tra.2015.10.004

Chiu, H.-M. 2005. A location model for the allocation of the off-street parking facilities, Journal of the Eastern Asia Society for Transportation Studies 6: 1344-1353.

http://doi.org/10.11175/easts.6.1344

Dehnavi, H. K.; Rezvan, M. T.; Shirmohammadli, A.; Vallée, D. 2013. A solution for urban road selection and construction problem using simulation and goal programming: case study of the city of Isfahan, Transport Policy 29: 46-53. https://doi.org/10.1016/j.tranpol.2013.04.003 
Dirickx, Y. M. I.; Jennergren, L. P. 1975. An analysis of the parking situation in the downtown area of West Berlin, Transportation Research 9(1): 1-11. https://doi.org/10.1016/0041-1647(75)90014-3

Drezner, Z.; Hamacher, H. 2002. Facility Location: Applications and Theory. Springer-Verlag Berlin Heidelberg. $460 \mathrm{p}$.

Ehrgott, M. 2005. Multicriteria Optimization. Springer. 323 p. https://doi.org/10.1007/3-540-27659-9

Eskandari, M. 2012. Off-Street Parking Facilities Location on the Urban Transportation Network with Multi Objectives (Case Study: Central District of Isfahan): MSc Thesis. Dept of Industrial and Systems Engineering, Isfahan University of technology, Iran.

Figueira, J.; Greco, S.; Ehrogott, M. 2005. Multiple Criteria Decision Analysis: State of the Art Surveys. Springer. 1048 p. https://doi.org/10.1007/b100605

Ghanbari, S.; Ghazi Asgar, A. 2011. Evaluation of different methods of site selection in management of public parking construction in central business of Esfahan using GIS, Geography and Environmental Planning 22(2): 37-40.

Goyal, S. K.; Gomes, L. F. A. M. 1984. A model for allocating car parking spaces in universities, Transportation Research Part B: Methodological 18(3): 267-269. https://doi.org/10.1016/0191-2615(84)90037-7

He, R.; Ma, C.; Li, Y. 2015. Site selection of public parking lot in the new district, International Journal of Wireless and Mobile Computing 8(2): 147-152. https://doi.org/10.1504/IJWMC.2015.068625

Jelokhani-Niaraki, M.; Malczewski, J. 2015a. A group multicriteria spatial decision support system for parking site selection problem: a case study, Land Use Policy 42: 492-508. https://doi.org/10.1016/j.landusepol.2014.09.003

Jelokhani-Niaraki, M.; Malczewski, J. 2015b. Decision complexity and consensus in Web-based spatial decision making: a case study of site selection problem using GIS and multicriteria analysis, Cities 45: 60-70. https://doi.org/10.1016/j.cities.2015.03.007

Khakbaz, A.; Nookabadi, A. S.; Shetab-Bushehri, S. N. 2013. A model for locating park-and-ride facilities on urban networks based on maximizing flow capture: a case study of Isfahan, Iran, Networks and Spatial Economics 13(1): 43-66. https://doi.org/10.1007/s11067-012-9172-4

Mavrotas, G. 2009. Effective implementation of the $\varepsilon$-constraint method in multi-objective mathematical programming problems, Applied Mathematics and Computation 213(2): 455-465. https://doi.org/10.1016/j.amc.2009.03.037

Naghsh e Jahan-Pars. 2008. Detailed Development Plan of Isfahan, Rules and Regulations of Planning and Building. 1st edition. Naghsh e Jahan-Pars: Consulting Engineers, Iran (in Persian).

Nosoohi, I.; Hejazi, S. R. 2011. A multi-objective approach to simultaneous determination of spare part numbers and preventive replacement times, Applied Mathematical Modelling 35(3): 1157-1166.

https://doi.org/10.1016/j.apm.2010.08.003

Rosenthal, R E. 2017. GAMS - A User's Guide. GAMS Development Corporation, Washington, DC, US. 588 p. Available from Internet: https://www.gams.com/latest/docs/userguides/GAMSUsersGuide.pdf

Shahi, J. 2011. Traffic Engineering. 11th edition, Center for Academic Publication, Tehran, Iran. (in Persian).
Tang, M.; Wang, X.; Yuan, S. 2013. Site selection of mechanical parking system based on GIS with AFRARBMI, Telkomnika: Indonesian Journal of Electrical Engineering and Computer Science (IJEECS) 11(7): 3935-3944.

Tehran Barnamerizi Shahri. 2006. Management Studies of Public Parking in Isfahan City. Final Report. Iran. (in Persian).

Tzeng, G.-H.; Huang, J.-J. 2011. Multiple Attribute Decision Making: Methods and Applications. Chapman and Hall/ CRC. 352 p.

Wang, Z.; Tan, Z.; Xu, H. 2008. Location model and algorithm of public parking facilities, in 2008 International Conference on Intelligent Computation Technology and Automation (ICICTA), 20-22 October 2008, Hunan, China. https://doi.org/10.1109/ICICTA.2008.368

Wey, W.-M. 2003. Dynamic parking facility location with timedependent demands: the progressive p-median problem, Proceedings of the Eastern Asia Society for Transportation Studies 4: 461-469. 